\title{
WELL-DISTRIBUTED SEQUENCES WITH RESPECT TO SYSTEMS OF CONVEX SETS
}

\section{H. NIEDERREITER ${ }^{1}$}

ABSTRACT. A theorem of W. M. Schmidt concerning the existence of sequences which are extremely well distributed with respect to suitable convex sets is generalized. We prove the existence of sequences which are simultaneously well distributed with respect to suitable systems of convex sets. The proof depends on combinatorial results dealing with the distribution of sequences in finite and countable sets.

1. Introduction. For $s \geq 2$, let $\bigcup^{s}=\left\{\left(u_{1}, \cdots, u_{s}\right) \in \mathbf{R}^{s}: 0 \leq u_{i}<1\right.$ for $1 \leq i \leq s\}$ be the $s$-dimensional half-open unit cube. We consider a sequence $x_{1}, x_{2}, \cdots$ of points in $U^{s}$. For a positive integer $n$ and a Lebesgue measurable subset $S$ of $\bigcup^{s}$, let $Z(n, S)$ be the number of $r, 1 \leq$ $r \leq n$, with $\mathbf{x}_{r} \in S$. We define the local discrepancy $D(n, S)=\mid Z(n, S)-$ $n \lambda(S) \mid$, where $\lambda$ denotes the $s$-dimensional Lebesgue measure, and $E(S)=$ $\sup _{n} D(n, S)$. In a recent paper, W. M. Schmidt has shown the following remarkable theorem which is connected with the notion of isotropic discrepancy (see [1, Chapter 2]) and results on irregularities of distribution in [6], [7], [8].

Theorem 1 (Schmidt [8]). For any $s \geq 2$, there exists a sequence $x_{1}$, $\mathbf{x}_{2}, \cdots$ in $\bigcup^{s}$ such that for every $\mu$ with $0 \leq \mu \leq 1$ there is a convex sub. set $S$ of $\bigcup^{s}$ satisfying $\lambda(S)=\mu$ and $E(S) \leq 1 / 2$.

In this note, we prove a generalization of Theorem 1 to systems of convex sets. The basic idea is to combine Schmidt's method with some deep results in combinatorial theory pertaining to the distribution of sequences in finite and countable sets. Our final result is as follows.

Theorem 2. For any $s \geq 2$, there exists a sequence $x_{1}, x_{2}, \cdots$ in $\bigcup^{s}$

Received by the editors July 31, 1973.

AMS (MOS) subject classifications (1970). Primary 10K 30; Secondary 10F 40, 05A99.

Key words and phrases. Isotropic discrepancy, irregularities of distribution, distribution in finite sets.

1 This research was carried out while the author was a participant of the 1973 Summer Research Institute in Number Theory at the University of Michigan. 
satisfying the following property: for every integer $k \geq 2$ and any numbers $\mu_{1}, \cdots, \mu_{k}$ in $[0,1]$ with $\sum_{j=1}^{k} \mu_{j}=1$, and also for any sequence $\mu_{1}, \mu_{2}$, $\cdots$ of numbers in $[0,1]$ with $\sum_{j=1}^{\infty} \mu_{j}=1$, there are convex subsets $S_{1}, \cdots$, $s_{k}$ (resp. $\left.s_{1}, s_{2}, \cdots\right)$ of $\bigcup^{s}$ with $\lambda\left(S_{j}\right)=\mu_{j}$ for $1 \leq j \leq k($ resp. $1 \leq j<\infty)$ and $E\left(S_{j}\right) \leq 1-1 /(2 k-2)$ for $1 \leq j \leq k$ (resp. $E\left(S_{j}\right) \leq 1$ for $\left.1 \leq j<\infty\right)$, and such that every point $\mathbf{x}_{n}$ of the sequence lies in a unique $S_{j}$.

We note that the case $k=2$ in Theorem 2 yields Theorem 1 (see also Remark 2). To avoid a trivial case, we remark that if $\mu_{j}=0$ for some $j$, we may take the corresponding $S_{j}$ to be the empty set. Thus, in the sequel, we can assume that $0<\mu_{j}<1$ for all $j$.

2. Some combinatorial lemmas. In this section, we collect some u seful facts concerning the distribution of sequences in finite and countable sets. This subject was studied recently in a number of papers [2], [3], [4], [5], [9].

For $k \geq 1$, let $j_{1}, j_{2}, \cdots$ be a sequence of elements from the set $\mathbf{Z}_{k}=$ $\{1,2, \cdots, k\}$. Given integers $n \geq 1$ and $j \in \mathbf{Z}_{k}$, we define the counting function $A(n, j)$ to be the number of $r, 1 \leq r \leq n$, with $j_{r}=j$.

Lemma $\mathbf{l}_{k}$ (Meijer [2]). For any $k \geq 2$ and any numbers $\mu_{1}, \cdots, \mu_{k}$ in $(0,1)$ with $\sum_{j=1}^{k} \mu_{j}=1$, there exists a sequence $j_{1}, j_{2}, \cdots$ in $\mathbf{Z}_{\mathbf{k}}$ satisfying $\left|A(n, j)-n \mu_{j}\right| \leq 1-1 /(2 k-2)$ for all $n \geq 1$ and all $j \in \mathbf{Z}_{k}$.

For a sequence $j_{1}, j_{2}, \cdots$ of elements from the set $\mathbf{Z}_{\infty}=\{1,2, \cdots\}$, we define the counting function $A(n, j)$ in a like manner as above.

Lemma $1_{\infty}$ (Tijdeman [9]). For any sequence $\mu_{1}, \mu_{2}, \cdots$ of numbers in $(0,1)$ with $\sum_{j=1}^{\infty} \mu_{j}=1$, there exists a sequence $j_{1}, j_{2}, \cdots$ in $\mathbf{Z}_{\infty}$ satisfying $\left|A(n, j)-n \mu_{j}\right| \leq 1$ for all $n \geq 1$ and $j \geq 1$.

To combine the two cases, we write $\epsilon_{k}=1 /(2 k-2)$ for $2 \leq k<\infty$ and $\epsilon_{\infty}=0$. In order to unify the discussion, $k$ may also attain the value $\infty$ from now on. We need a simple auxiliary result complementing the above two lemmas in the case of some $\mu_{j}$ being close to 1 .

Lemma 2. Let $2 \leq k \leq \infty$, let the $\mu_{j}, j \in \mathbf{Z}_{k}$, be as in Lemma $1_{k}$, and suppose that $1 / 2<\mu_{h}<1$ for some $h \in \mathbf{Z}_{k}$. Let $m$ be the largest integer such that $\mu_{h}>1-1 / m$. Then there exists a sequence $j_{1}, j_{2}, \cdots$ in $\mathbf{Z}_{k}$ with $j_{r}=h$ for $1 \leq r \leq[\mathrm{m} / 2]$ and $\left|A(n, j)-n \mu_{j}\right| \leq 1-\epsilon_{k}$ for all $n \geq 1$ and all $j \in \mathbf{Z}_{k}$.

Proof. According to Lemma $1_{k}$, there exists a sequence $i_{1}, i_{2}, \cdots$ in 
$\mathbf{Z}_{k}$ with $\left|A(n, j)-n \mu_{j}\right| \leq 1-\epsilon_{k}$ for all $n \geq 1$ and all $j \in \mathbf{Z}_{k}$. In particular, we have

$$
A([m / 2]+1, b) \geq([m / 2]+1) \mu_{h}-1+\epsilon_{k} .
$$

Using $m \geq 2$, it follows that

$$
\begin{aligned}
A([m / 2]+1, b) & >([m / 2]+1)(1-1 / m)-1+\epsilon_{k} \\
& \geq[m / 2]-1+\epsilon_{k} \geq[m / 2]-1 .
\end{aligned}
$$

Since $A([m / 2]+1, h)$ is an integer, we arrive at $A([m / 2]+1, h) \geq[m / 2]$. Thus, at most one of the $i_{r}$ with $1 \leq r \leq[\mathrm{m} / 2]+1$ can be different from $h$. It remains to consider the case that $i_{r} \neq h$ for some $r$ with $1 \leq r \leq[\mathrm{m} / 2]$. We define a new sequence $j_{1}, j_{2}, \cdots$ in $\mathbf{Z}_{k}$ by setting $j_{n}=h$ for $1 \leq n \leq$ $[\mathrm{m} / 2], j_{n}=i_{r}$ for $n=[\mathrm{m} / 2]+1$, and $j_{n}=i_{n}$ for $n>[\mathrm{m} / 2]+1$. For $1 \leq n \leq$ $r-1$, and also for $n \geq[m / 2]+1$, the counting functions $A(n, j)$ of the old and the new sequence are identical. For $r \leq n \leq[\mathrm{m} / 2]$, the counting function of the new sequence satisfies

$$
\left|A(n, b)-n \mu_{h}\right|=n\left(1-\mu_{h}\right)<n / m \leq 1 / 2 \leq 1-\epsilon_{k},
$$

and

$$
\left|A(n, j)-n \mu_{j}\right|=n \mu_{j}<n / m \leq 1-\epsilon_{k}
$$

for $j \neq h$, since in this case $\mu_{j}<1 / m$. Therefore the new sequence enjoys all the required properties.

3. Proof of Theorem 2. We observe that it suffices to prove the theorem for $s=2$. For if $\mathbf{x}_{1}=\left(x_{1}, y_{1}\right), \mathbf{x}_{2}=\left(x_{2}, y_{2}\right), \cdots$ is a sequence in $U^{2}$ serving the desired purpose, then for $s \geq 3$ the sequence $x_{1}^{\prime}, x_{2}^{\prime}, \cdots$ in $\bigcup^{s}$ defined by $\mathbf{x}_{n}^{\prime}=\left(x_{n}, y_{n}, 0, \cdots, 0\right)$ for $n \geq 1$ will do. To see this, one just has to take for any $k, 2 \leq k \leq \infty$, the convex subsets $s_{j}, j \in \mathbf{Z}_{k}$, of $\bigcup^{2}$ associated with a possible choice of numbers $\mu_{j}, j \in \mathbf{Z}_{k}$, according to Theorem 2 , and to replace them by the convex subsets $S_{j}^{\prime}=s_{j} \times \bigcup^{s-2}, j \in \mathbf{Z}_{k}$, of $\bigcup^{s}$.

We consider now the case $s=2$. Let $t_{1}, t_{2}, \cdots$ be a decreasing sequence of real numbers with $0<t_{n} \leq 1 /(8 n)$ for $n \geq 1$, and put

$$
\mathbf{x}_{n}=\left(x_{n}, y_{n}\right)=\left(1-\cos t_{n}, \sin t_{n}\right)
$$

for $n \geq 1$. All the points $x_{n}$ lie on the circle $(x-1)^{2}+y^{2}=1$. For a given $k, 2 \leq k \leq \infty$, let the $\mu_{j}, j \in \mathbf{Z}_{k}$, be numbers in the interval $(0,1)$ with $\Sigma_{j \in Z_{k}} \mu_{j}=1$. According to Lemma $1_{k}$, there exists a sequence $j_{1}, j_{2}, \cdots$ 
in $\mathbf{Z}_{k}$ with $\left|A(n, j)-n \mu_{j}\right| \leq 1-\epsilon_{k}$ for all $n \geq 1$ and all $j \in \mathbf{Z}_{k}$. In case $1 / 2<\mu_{h}<1$ for some $h \in \mathbf{Z}_{k}$, we suppose al so that the sequence $j_{1}, j_{2}, \cdots$ satisfies the additional condition in Lemma 2. For $j \in \mathbf{Z}_{k}$ and $n \geq 1$, let $F(n, j)$ be the set of those $\mathbf{x}_{i}, 1 \leq i \leq n$, with $j_{i}=j$. We have of course $\operatorname{card} F(n, j)=A(n, j)$, and therefore

$$
\mid \text { card } F(n, j)-n \mu_{j} \mid \leq 1-\epsilon_{k} \text { for all } n \geq 1 \text { and all } j \in \mathbf{Z}_{k} \text {. }
$$

Moreover, the sets $F(n, j)$ and $F\left(n^{\prime}, j^{\prime}\right)$ are disjoint as soon as $j \neq j^{\prime}$.

For fixed $j \in \mathbf{Z}_{k}$, we define a convex subset $G_{1}(j)$ of $\bigcup^{2}$ by letting $G_{1}(j)$ be the convex hull of the points $\mathbf{x}_{i}$ appearing in some $F(n, j)$. It follows from (1) that $\lim _{n \rightarrow \infty}$ card $F(n, j)=\infty$. In addition, the sequence card $F(n, j), n=1,2, \cdots$, is nondecreasing and attains all positive integers as values. Let $n_{2}$ be the smallest positive integer with card $F\left(n_{2}, j\right)$ $=2$. Then there exists $n_{1}, 1 \leq n_{1}<n_{2}$, such that $\mathbf{x}_{n_{1}}, \mathbf{x}_{n_{2}} \in F\left(n_{2}, j\right) \subseteq$ $G_{1}(j)$. It is easily seen that $\lambda\left(G_{1}(j)\right)$ is at most the area of the triangle bounded by the $y$-axis, the line segment joining $x_{n_{1}}$ and the origin, and the line joining $x_{n_{1}}$ and $x_{n_{2}}$. Hence we get

$$
\lambda\left(G_{1}(j)\right) \leq 1 / 2 y_{n_{2}}=1 / 2 \sin t_{n_{2}}<1 / 2 t_{n_{2}} \leq 1 /\left(16 n_{2}\right) .
$$

On the other hand, it follows from (1) with $n=n_{2}$ that $\left|2-n_{2} \mu_{j}\right| \leq 1-\epsilon_{k}$, and so $n_{2} \mu_{j} \geq 1$. Therefore $\lambda\left(G_{1}(j)\right) \leq \mu_{j} / 16<\mu_{j}$.

For fixed $j \in \mathbf{Z}_{k}$, we define a convex subset $G_{2}(j)$ of $\bigcup^{2}$ as follows. If $0<\mu_{j} \leq 1 / 2$, let $G_{2}(j)$ be the convex hull of the triangle $0 \leq x<1,0 \leq$ $y<1, y<x$, and of the points $\mathbf{x}_{i}$ appearing in some $F(n, j)$. Then $\lambda\left(G_{2}(j)\right)$ $>1 / 2 \geq \mu_{j}$. If $1 / 2<\mu_{j}<1$, let $m$ be the largest integer such that $\mu_{j}>1-1 / m$. Then $G_{2}(j)$ is taken to be the convex hull of the points $\mathbf{x}_{i}$ appearing in some $F(n, j)$ and of the open quadrilateral with vertices $\mathbf{x}_{[m / 2]},(1,0)$, $(1,1)$, and $\mathbf{x}^{*}$, where $\mathbf{x}^{*}=\left(x^{*}, 1\right)$ is the intersection of the line $y=1$ and the tangent to the circle $(x-1)^{2}+y^{2}=1$ at $x_{[m / 2]}$. The quadrilateral contains the open rectangle with vertices $\left(x^{*}, y_{[\mathrm{m} / 2]}\right),\left(1, y_{[\mathrm{m} / 2]}\right),(1,1)$, and $\left(x^{*}, 1\right)$ of area

$$
\left(1-x^{*}\right)\left(1-y_{[m / 2]}\right)=\left(1-y_{[m / 2]}\right)^{2}\left(1-x_{[m / 2]}\right)^{-1} .
$$

Therefore

$$
\begin{aligned}
\lambda\left(G_{2}(j)\right) & \left.>\left(1-y_{[m / 2]}\right)^{2}>1-2 y_{[m / 2]}=1-2 \sin t_{[m / 2}\right] \\
& >1-2 t_{[m / 2]} \geq 1-1 /(4[m / 2]) \geq 1-1 /(m+1) \geq \mu_{j},
\end{aligned}
$$


since $4[m / 2] \geq m+1$ for $m \geq 2$. Thus in both cases we have $\lambda\left(G_{2}(j)\right)>\mu_{j}$.

The proof is completed as in [8]. By construction, we have $G_{1}(j) \subseteq$ $G_{2}(j)$, and it was established above that $\lambda\left(G_{1}(j)\right)<\mu_{j}<\lambda\left(G_{2}(j)\right)$. There exists a convex subset $S_{j}$ of $\bigcup^{2}$ with $G_{1}(j) \subseteq S_{j} \subseteq G_{2}(j)$ and $\lambda\left(S_{j}\right)=\mu_{j}$. Again by construction, we have

$$
G_{1}(j) \cap\left\{\mathbf{x}_{1}, \cdots, \mathbf{x}_{n}\right\}=G_{2}(j) \cap\left\{\mathbf{x}_{1}, \cdots, \mathbf{x}_{n}\right\}=F(n, j)
$$

for all $n \geq 1$; therefore $S_{j} \cap\left\{\mathbf{x}_{1}, \cdots, \mathbf{x}_{n}\right\}=F(n, j)$ for all $n \geq 1$. By (1), we get

$$
D\left(n, S_{j}\right)=\left|Z\left(n, S_{j}\right)-n \lambda\left(S_{j}\right)\right|=\left|c \operatorname{ard} F(n, j)-n \mu_{j}\right| \leq 1-\epsilon_{k}
$$

for all $n \geq 1$, and so $E\left(S_{j}\right) \leq 1-\epsilon_{k}$. From the definition of the sets $F(n, j)$, it follows that every $\mathbf{x}_{n}$ lies in at least one $S_{j}$. Furthermore, for $i, j \in \mathbf{Z}_{k}$ with $i \neq j$, the intersection $S_{i} \cap S_{j}$ cannot contain any $\mathbf{x}_{n}$, for otherwise $F(n, i) \cap F(n, j)$ would be nonempty for some $n \geq 1$.

4. Concluding remarks. To what extent is Theorem 2 best possible? We add some remarks concerning this question and related matters.

Remark 1. The constant $1-\epsilon_{k}$ in Theorem 2 is best possible. For suppose there exists $\delta_{k}>\epsilon_{k}$ such that Theorem 2 holds with $E\left(S_{j}\right) \leq 1-\epsilon_{k}$ replaced by $E\left(S_{j}\right) \leq 1-\delta_{k}$. We note that for every $n \geq 1$ there exists a unique $j_{n} \in \mathbf{Z}_{k}$ with $\mathbf{x}_{n} \in S_{j_{n}}$. Consider the sequence $j_{1}, j_{2}, \cdots$ in $\mathbf{Z}_{k}$. We have $A(n, j)=Z\left(n, S_{j}\right)$ for all $n \geq 1$ and all $j \in \mathbf{Z}_{k}$, and so

$$
\left|A(n, j)-n \mu_{j}\right|=\left|Z\left(n, S_{j}\right)-n \lambda\left(S_{j}\right)\right| \leq 1-\delta_{k} \text {. }
$$

Thus for any choice of numbers $\mu_{j}, j \in \mathbf{Z}_{k}$, in $[0,1]$ with $\Sigma_{j \in \mathbf{Z}_{k}} \mu_{j}=1$, there would exist a sequence in $\mathbf{Z}_{k}$ satisfying $\left|A(n, j)-n \mu_{j}\right| \leq 1-\delta_{k}$ for all $n \geq 1$ and $j \in \mathbf{Z}_{k}$. However, this contradicts a result of Tijdeman [9].

Remark 2. If $1 \leq k \leq \infty$ and the $\mu_{j}, j \in \mathbf{Z}_{k}$, are numbers in $[0,1]$ with $\Sigma_{j \in Z_{k}} \mu_{j}<1$, one may introduce the number $\mu_{0}=1-\Sigma_{j \in Z_{k}} \mu_{j}$ and apply Theorem 2. One arrives at a result analogous to Theorem 2 , with the bounds on the $E\left(S_{j}\right)$ being $1-1 /(2 k)$ for finite $k$ and 1 for $k=\infty$, and with the last condition replaced by the following one: "every point $x_{n}$ of the sequence lies in at most one $S_{j}$ ".

Remark 3. If $2 \leq k \leq \infty$ and the $\mu_{j}, j \in \mathbf{Z}_{k}$, are numbers in $[0,1]$ with $\Sigma_{j \in \mathrm{Z}_{k}} \mu_{j}>1$, then a result analogous to Theorem 2 cannot hold, even if we allow the dependence of the sequence on the $\mu_{j}$ and relax the conditions on the $S_{j}$. For suppose $x_{1}, x_{2}, \cdots$ is a sequence in $U^{s}$ for which there exist measurable subsets $S_{j}, j \in \mathbf{Z}_{k}$, of $\bigcup^{s}$ with $\lambda\left(S_{j}\right)=\mu_{j}$ and $E\left(S_{j}\right) \leq$ 
$B_{k}$ for all $j \in \mathbf{Z}_{k}$ and some finite $B_{k}$, and such that every $\mathbf{x}_{n}$ lies in a unique $S_{j}$. Using the same arguments as in Remark 1 , we arrive at a sequence in $\mathbf{Z}_{k}$ satisfying $\left|A(n, j)-n \mu_{j}\right| \leq B_{k}$ for all $n \geq 1$ and all $j \in \mathbf{Z}_{k}$. It follows that $\lim _{n \rightarrow \infty} A(n, j) / n=\mu_{j}$. On the other hand, by choosing $p \in \mathbf{Z}_{k}$ with $\sum_{j=1}^{p} \mu_{j}>1$, we get

$$
1 \geq \lim _{n \rightarrow \infty} \sum_{j=1}^{p} \frac{A(n, j)}{n}=\sum_{j=1}^{p} \mu_{j}>1,
$$

a contradiction.

Remark 4. If the sequence $x_{1}, x_{2}, \cdots$ in Theorem 2 may depend on $k$ and the numbers $\mu_{j}, j \in \mathbf{Z}_{k}$, a much simpler construction can be given. For each $i \in \mathbf{Z}_{k}$, define $\lambda_{i}=\Sigma_{j=1}^{i} \mu_{j}$ and $S_{i}=\left[\lambda_{i-1}, \lambda_{i}\right) \times \bigcup^{s-1}$ (with $\lambda_{0}=0$ ). Choose a point $\mathbf{y}_{i} \in S_{i}$. Let $j_{1}, j_{2}, \cdots$ be a sequence in $\mathbf{Z}_{k}$ satisfying the property in Lemma $1_{k}$. Then for the sequence $x_{1}, x_{2}, \cdots$ in $\bigcup^{s}$ with $\mathbf{x}_{n}=\mathbf{y}_{j}, n \geq 1$, the intervals $S_{i}, i \in \mathbf{Z}_{k}$, meet all the requirements of Theorem $2^{n}$

\section{REFERENCES}

1. L. Kuipers and H. Niederreiter, Uniform distribution of sequences, Wiley, New York, 1974.

2. H. G. Meijer, On a distribution problem in finite sets, Indag. Math. 35 (1973), $9-17$.

3. H. G. Meijer and H. Niederreiter, On a distribution problem in finite sets, Compositio Math. 25 (1972), 153-160.

4. H. Niederreiter, On the existence of uniformly distributed sequences in compact spaces, Compositio Math. 25 (1972), 93-99.

5. - A distribution problem in finite sets, Applications of Number Theory to Numerical Analysis, S. K. Zaremba (Editor), Academic Press, New York, 1972, pp. 237-248.

6. W. M. Schmidt, Irregularities of distribution. II, Trans. Amer. Math. Soc. 136 (1969), 347-360. MR 38 \#3237.

7. - Irregularities of distribution. IV, Invent. Math. 7 (1969), 55-82. MR $39 \# 6838$.

8. - Irregularities of distribution. VII, Trans. Amer. Math. Soc. 198 (1974), 1-22.

9. R. Tijdeman, On a distribution problem in finite and countable sets, J. Combinatorial Theory Ser. A 15 (1973), 129-137.

DEPARTMENT OF MATHEMATICS, SOUTHERN ILLINOIS UNIVERSITY, CARBONDALE, ILLINOIS 62901

Current address: School of Mathematics, Institute for Advanced Study, Princeton, New Jersey 08540 